\title{
Modulation of Resting-State Amygdala-Frontal Functional Connectivity by Oxytocin in Generalized Social Anxiety Disorder
}

\author{
Sonam Dodhia ${ }^{1,9}$, Avinash Hosanagar ${ }^{2,3,9}$, Daniel A Fitzgerald ${ }^{1,7}$, Izelle Labuschagne ${ }^{4}$, Amanda G Wood ${ }^{4,5}$, \\ Pradeep J Nathan ${ }^{4,6}$ and K Luan Phan*, , , , 8 \\ 'Department of Psychiatry, University of Illinois at Chicago, Chicago, IL, USA; '2Department of Psychiatry, University of Michigan, Ann Arbor, MI, \\ USA; ${ }^{3}$ Mental Health Service, VA Ann Arbor Healthcare System, Ann Arbor, MI, USA; ${ }^{4}$ School of Psychological Sciences, Monash University, \\ Melbourne, Victoria, Australia; ${ }^{5}$ School of Psychology, University of Birmingham, Birmingham, UK; ${ }^{6}$ Department of Psychiatry, Cambridge \\ University, Cambridge, UK; ${ }^{7}$ Mental Health Service Line, Jesse Brown VA Medical Center, Chicago, IL, USA; ${ }^{8}$ Departments of Psychology and \\ Anatomy and Cell Biology, University of Illinois at Chicago, Chicago, IL, USA
}

\begin{abstract}
Generalized social anxiety disorder (GSAD) is characterized by aberrant patterns of amygdala-frontal connectivity to social signals of threat and at rest. The neuropeptide oxytocin (OXT) modulates anxiety, stress, and social behaviors. Recent functional neuroimaging studies suggest that these effects are mediated through OXT's effects on amygdala reactivity and/or amygdala-frontal connectivity. The aim of the current study was to examine OXT's effects on amygdala-frontal resting-state functional connectivity (rsFC) in GSAD patients and healthy controls (HCs). In a randomized, double-blind, cross-over design, 18 GSAD and I 8 HC participants received intranasal OXT $(24 \mathrm{IU}$ or $40.32 \mu \mathrm{g})$ or placebo (PBO) before resting-state functional magnetic resonance imaging. In individuals with GSAD, OXT enhanced rsFC of the left and right amygdala with rostral anterior cingulate cortex (ACC)/medial prefrontal cortex (mPFC), and in doing so, reversed (ie, 'normalized') the reduced amygdala-frontal connectivity observed relative to HCs evident on PBO. Higher social anxiety severity in GSAD subjects correlated with lower amygdala-ACC/mPFC connectivity on PBO and higher social anxiety also correlated with greater enhancement in amygdala-frontal connectivity induced by OXT. These findings show that OXT modulates a neural circuit known for social threat processing and emotion regulation, suggesting a neural mechanism by which OXT may have a role in the pathophysiology and treatment of social anxiety disorder.

Neuropsychopharmacology (20I4) 39, 206 I-2069; doi:I0.I038/npp.20I4.53; published online 2 April 2014
\end{abstract}

\section{INTRODUCTION}

Oxytocin (OXT), a neuropeptide produced in the hypothalamus, has been shown to have a prosocial effect on behavior along with benefits on social cognition (Bartz et al, 2011). The social functions of OXT have been demonstrated in various animal studies including its role in social memory (Bielsky and Young, 2004), stress, and anxiety (Carter et al, 2008). In humans, OXT has been demonstrated to have a role in social memory, emotion perception, and empathy (Bartz et al, 2011). This is consistent with the distribution of OXT receptors in areas of the brain associated with social and anxiety-related behaviors such as the amygdala (Gimpl and Fahrenholz, 2001). While the exact mechanism of OXT's action is unknown, it has been

*Correspondence: Professor KL Phan, Department of Psychiatry, University of Illinois at Chicago, 1747 West Roosevelt Road, IJR WROB Suite 244, Chicago, IL 60608, USA, Tel: +3I2 355 5954,

E-mail: klphan@uic.edu

${ }^{9}$ Designates equal contribution as first authors.

Received 31 October 2013; revised 19 February 2014; accepted 21 February 2014; accepted article preview online 5 March 2014 postulated to exert an inhibitory effect via activation of GABAergic interneurons within the amygdala (Ehrlich et al, 2009; Huber et al, 2005). In keeping with this hypothesis, imaging studies in healthy human subjects have shown that OXT attenuates amygdala response to faces showing anger (Domes et al, 2007) and to fear conditioned socially relevant faces (Petrovic et al, 2008).

Generalized social anxiety disorder (GSAD) is a common psychiatric illness with exaggerated and pervasive fear of negative scrutiny by others during social situations. It is associated with significant psychiatric comorbidity, occupational, and social impairment (Stein and Stein, 2008). This disorder is characterized by an exaggerated fear response during the anticipation and perception of social evaluative threat (Davidson et al, 2000; Phan et al, 2006). Functional magnetic resonance imaging (MRI) studies have consistently demonstrated amygdala hyperactivity in responses to cues conveying threat in subjects with GSAD (Evans et al, 2008; Goldin et al, 2009; Phan et al, 2006; Stein et al, 2002). The amygdala is anatomically and functionally connected to areas of the frontal cortex, especially medial prefrontal cortex (mPFC) (Ghashghaei et al, 2007; Kim et al, 2011) 
which is an area considered as a critical node in the brain's social cognitive network (Adolphs, 2009). Along with amygdala hyperactivity, GSAD patients have also been demonstrated to show exaggerated $\mathrm{mPFC}$ and anterior cingulate cortex (ACC) activation in functional imaging studies (Amir et al, 2005; Phan et al, 2006).

Convergent evidence suggests that OXT may have a key role in disorders with social dysfunction including GSAD and depression (Zink and Meyer-Lindenberg, 2012). Recent neuroimaging studies looking at OXT effects in GSAD patients found that, relative to healthy normal controls, OXT compared with placebo (PBO) normalized the amygdala hyperactivation elicited by fearful social stimuli (Labuschagne et al, 2010). Similarly, OXT was also found to reduce the heightened activation in the mPFC/ACC regions in GSAD while processing sad faces (Labuschagne et al, 2011).

Advances in neuroimaging techniques have permitted the examination of amygdala-frontal networks at rest. In the absence of an overt task, resting-state functional MRI (rsFMRI) permits the study of brain networks at baseline and may allow for interpretation of a more pervasive deficit. rsFMRI studies in GSAD patients have shown abnormal amygdala-mPFC and amygdala-cingulate connectivity (Liao et al, 2010; Prater et al, 2013). While previous neuroimaging studies have primarily studied the effects of OXT on activation of the amygdala, a limited number of studies looking at effects of OXT on connectivity exist in the literature. In a recent resting-state functional connectivity (rsFC) study, Sripada et al (2013) demonstrated that OXT enhanced connectivity between bilateral amygdala and mPFC in healthy controls (HCs). Understanding the effects of OXT on amygdala-cortical connectivity in GSAD would therefore add to our understanding of the underlying mechanisms of OXT's central effects (Bethlehem et al, 2013) and by which OXT may have a role in the pathophysiology and/or treatment of GSAD.

The aim of this study was to explore the effects of OXT on amygdala-frontal rsFC in GSAD patients and HCs. In a randomized, double-blind $\mathrm{PBO}-$ controlled, cross-over design, 36 (18 healthy and 18 GSAD) male participants received OXT or PBO $45 \mathrm{~min}$ before rsFMRI. Given prior evidence that amygdala and $\mathrm{mPFC} / \mathrm{ACC}$ connectivity is disrupted in GSAD, and that OXT reduced hyperactivation in the amygdala and mPFC, we hypothesized that OXT would 'normalize' or reverse the pattern of altered amygdalamPFC/ACC connectivity in GSAD, such that the GSAD group would exhibit reduced amygdala-mPFC/ACC at baseline (under $\mathrm{PBO}$ ) relative to the control group, and show enhanced amygdala-mPFC/ACC connectivity on OXT.

\section{MATERIALS AND METHODS}

\section{Subjects}

This study included 18 right-handed males $(29.9 \pm 10.2$ years) with GSAD and 18 right-handed males (mean age $29.4 \pm 9.0$ years) who were HCs (Table 1). The participants' age range was $19-55$ years. Data from one HC participant were excluded due to poor quality of data collected. A GSAD diagnosis was determined by the Clinical International Diagnostic Interview (CIDI) version 2.1 (WHO, 1997) and the Liebowitz Social Anxiety Scale (LSAS) total score
Table I Demographic and Clinical Characteristics

\begin{tabular}{|c|c|c|c|c|}
\hline & $\mathrm{HC}$ & GSAD & $t$-score ${ }^{a}$ & $p$-Value \\
\hline Age & $29.89(10.2)$ & $29.39(9.0)$ & 0.156 & 0.877 \\
\hline Education & $16.00(2.5)$ & $14.67(1.6)$ & 1.930 & 0.062 \\
\hline BDI-II & $1.28(1.9)$ & $10.83(7.5)$ & -5.265 & $<0.001$ \\
\hline BAl & $2.17(5.0)$ & I $6.89(8.2)$ & -6.503 & $<0.001$ \\
\hline State anxiety (STAI) & $23.59(7.0)$ & $38.8 \mid(\mid 5.8)$ & -6.135 & $<0.001$ \\
\hline Trait anxiety $(\mathrm{STAl})^{\mathrm{b}}$ & $27.35(8.2)$ & $50.44(11.5)$ & -7.785 & $<0.001$ \\
\hline LSAS: total & I3.94 (8.3) & $81.67(17.5)$ & -14.828 & $<0.001$ \\
\hline LSAS: performance & $6.89(5.1)$ & $38.11(11.0)$ & -10.916 & $<0.00$ । \\
\hline LSAS: social situations & $7.06(4.6)$ & $43.56(8.6)$ & -15.876 & $<0.001$ \\
\hline
\end{tabular}

Abbreviations: BAI, Beck Anxiety Inventory; BDI-II, Beck Depression Inventory version II; GSAD, Generalized Social Anxiety Disorder subjects; HC, healthy control subjects; LSAS, Liebowitz Social Anxiety Scale; STAI, State-Trait Anxiety Inventory.

Data are expressed as mean ( $\pm \mathrm{SD}$; standard deviation); $n=18$ per group. ${ }^{a} t$-tests, two-tailed.

${ }^{b}$ Data missing from two GSAD $(n=16)$ and one HC $(n=17)$ subjects.

(Liebowitz, 1987), and the diagnosis was confirmed with a physician interview. The LSAS features 24 items, 13 relating to performance anxiety and 11 concerning social situations. GSAD subjects had an LSAS total score of $>70$ and a score of $>30$ on each subscale for performance and situational anxiety. The CIDI was used to confirm that no GSAD participant had another anxiety disorder (such as generalized anxiety disorder or panic disorder) that was more prominent than GSAD. GSAD subjects had not experienced a depressive episode in the 6 months before testing and had not abused alcohol or a substance in the year before testing. GSAD subjects also had no history of other psychiatric disorders such as bipolar disorder, post-traumatic stress disorder, and schizophrenia. All participants were non-smokers, were not on medication (and were not previously on medication), and did not have allergies. Participants also had not experienced head injury or neurological trauma that resulted in a loss of consciousness and did not have mental retardation. All participants were screened for other psychiatric disorders using the CIDI and anxiety and depression symptomatology was assessed with the Beck Depression Inventory (BDI-II; Beck et al, 1996), Beck Anxiety Inventory (BAI; Steer and Beck, 1997), and the State-Trait Anxiety Inventory (STAI; Spielberger, 1983) and also underwent a brief physical exam by a physician before testing. This study was approved by the Standing Committee on Ethics in Research involving Humans of Monash University and all the subjects provided written informed consent.

\section{Study Design and Drug Administration}

The current study was a within-subjects, randomized, double-blind, PBO-controlled, counterbalanced study. Each participant received two acute treatments separated by a 1-week wash-out period. The two treatments were intranasal sprays of OXT ( $24 \mathrm{IU}$ or $40.32 \mu \mathrm{g}$ ) or PBO (same ingredients as OXT minus the peptide). Each participant arrived for the treatment sessions $1 \mathrm{~h}$ before MRI scanning. Participants did not consume food $1 \mathrm{~h}$ before MRI scanning and did not 
consume caffeine or alcohol on the day of testing. At $45 \mathrm{~min}$ before the MRI scanning, each participant self-administered the treatment of either OXT or PBO. Each participant delivered the treatment to himself in portions of three puffs of $4 \mathrm{IU}$ or $6.72 \mu \mathrm{g}$ per nostril. The puffs were delivered to each nostril in an alternating order and with a 45-s break between each puff to each nostril. The MRI scanning commenced $45 \mathrm{~min}$ post treatment because this is the predicted optimal time necessary for OT's effects to be observed (Domes et al, 2007; Kirsch et al, 2005). The participants also performed two different emotional face processing tasks that typically occurred before the restingstate scan, one involving threat (angry/fearful) faces (Labuschagne et al, 2010) and another involving sad/happy faces (Labuschagne et al, 2011); these data were analyzed separately from the resting-state results and previously published. OXT effects on subjective mood were previously reported (Labuschagne et al, 2010).

\section{Resting-State fMRI}

Each subject was positioned supine in the MRI scanner with his head in a comfortable restraint to reduce movement. Subjects were instructed to keep their eyes closed, relax and let their minds wander, but not to fall sleep. The scan lasted $3 \min 20 \mathrm{~s}$.

\section{MRI Acquisition}

A 3T Siemens Tim Trio scanner with 12-channel head coil was used to perform MRI. Functional images employed a gradient-echo echo-planar imaging (EPI) sequence that is sensitive to blood oxygenated level-dependent (BOLD) contrast $\left(40 \mathrm{~ms}\right.$ TE, $2 \mathrm{~s}$ TR, $90^{\circ}$ flip, $210 \mathrm{~mm}$ FOV, $128 \times$ 128 matrix, 25 contiguous $5 \mathrm{~mm}$ slices parallel to the hippocampus and interleaved). We also acquired from all participants whole-brain T1-weighed anatomical reference images $\left(2.15 \mathrm{~ms}\right.$ TE, $1.9 \mathrm{~s}$ TR, $9^{\circ}$ flip, $256 \mathrm{~mm}$ FOV, 176 sagittal slices, $1 \mathrm{~mm}$ slice thickness, perpendicular to the anterior-posterior commissure line).

\section{Data Analysis}

Connectivity analyses were performed using Statistical Parametric Mapping software (SPM8: Wellcome Trust Centre for Neuroimaging, London, UK) and the Functional Connectivity (CONN) toolbox (Whitfield-Gabrieli and Nieto-Castanon, 2012). All subjects met minimum movement threshold of $<3 \mathrm{~mm}$ in any direction to be included in the analyses. Maximum total head movement and averaged scan-to-scan (ie, between scan) head motion was measured and compared between-sessions and between-groups to ensure motion did not differ between conditions (see below); averaged scan-to-scan head motion was incorporated as covariates in the fMRI analyses at the second level. The preprocessing of the images included slice-time correction, motor realignment, normalization, and smoothing. Using the component-based method, noise signals, such as cerebrospinal fluid, white matter, movement parameters, and time-series predictors of global signal, were removed from the images (Behzadi et al, 2007). In the seed-based analyses, temporal correlations of the resting-state BOLD signal time series were examined between the left and right amygdala seed regions (anatomically derived regions of interest/ROIs from the Automated Anatomical Labeling toolbox based on the atlas of Tzourio-Mazoyer et al (2002) and the rest of the brain. During first-level processing, an individual contrast image was created for each participant. During second-level processing, whole-brain voxel-wise repeated measures analyses of variance (ANOVAs) were performed in order to test for main effects of drug (OXT, $\mathrm{PBO}$ ), of group (GSAD, HC), and drug-by-group interactions. To test our main prediction that GSAD would exhibit reduced amygdala-mPFC/ACC at baseline (under $\mathrm{PBO}$ ) relative to controls and show enhanced amygdala-mPFC/ ACC connectivity on OXT, we focused on the results showing significant drug-by-group interactions; however, we also report significant main effects of drug and of group for completeness. To determine significance within a priori regions (mPFC, ACC) in which we had a strong hypothesis, we restricted our analyses to these relevant using an anatomically derived (AAL atlas) partial brain mask of entire prefrontal cortex/frontal lobe (search volume $=451$ $840 \mathrm{~mm}^{3}$, encompassing 56480 voxels). For the ANOVA of left and right amygdala connectivity, cluster-based significance thresholding was used to adjust for multiple comparisons within the search volume. On the basis of simulations (10000 iterations) and estimated smoothness (FWHM of $4.4 \mathrm{~mm} \times 5.6 \mathrm{~mm} \times 5.6 \mathrm{~mm}$ ) performed with 3 DClustSim (http://afni.nimh.nih.gov/pub/dist/doc/program_help/ $3 \mathrm{dClustSim.html),} \mathrm{a} \mathrm{family-wise} \mathrm{error} \mathrm{correction} \mathrm{at}$ $\alpha<0.05$ is achieved with a voxel threshold of $p<0.005$ and a cluster size of at least 44 voxels $\left(352 \mathrm{~mm}^{3}\right)$. For a priori areas showing drug-by-group interactions, we extracted parameter estimates/ $\beta$ weights (connectivity values, arbitrary units) from the entire cluster for each subject to conduct post hoc paired and independent samples $t$-tests and illustrate the direction of effects. To investigate the relationship between connectivity differences and clinical symptom severity, we conducted Pearson's correlation coefficient analyses to examine the relationship between amygdala connectivity and social anxiety symptom severity as measured by the total score of the LSAS (LSAS-Total) and given the predicted function of OXT 'prosocial' effects, the social situations subscore of the LSAS (LSAS-Social). Across the rest of the brain, for completeness to obviate bias and to generate new hypotheses, we show significant main effects and interactions at $p<0.005$ (uncorrected) and a minimum cluster extent threshold of 40 contiguous voxels (volume $=320 \mathrm{~mm}^{3}$ ).

\section{RESULTS}

A significant drug-by-group interaction in $\mathrm{rsFC}$ was observed between the left amygdala and the rostral ACC (Brodmann Area (BA) 32) (MNI peak [12, 46, 0], $F(1,65)=$ $11.16, p=0.001$ ), encompassing a cluster (volume $=1008$ $\mathrm{mm}^{3}$ ) extending anteriorly toward the rostral mPFC (BA 10, subpeak at $[4,50,2], F(1,65)=10.38, p=0.002$ ) (Figure 1a; Table 2). As predicted, post hoc t-tests revealed that: (1) GSAD patients exhibited reduced left amygdala-ACC/mPFC connectivity relative to $\mathrm{HCs}$ at baseline (on $\mathrm{PBO}$ ), $t(1,33)=-2.58, p=0.015$; (2) OXT (compared with $\mathrm{PBO})$ enhanced left amygdala-ACC/mPFC connectivity at a trend 

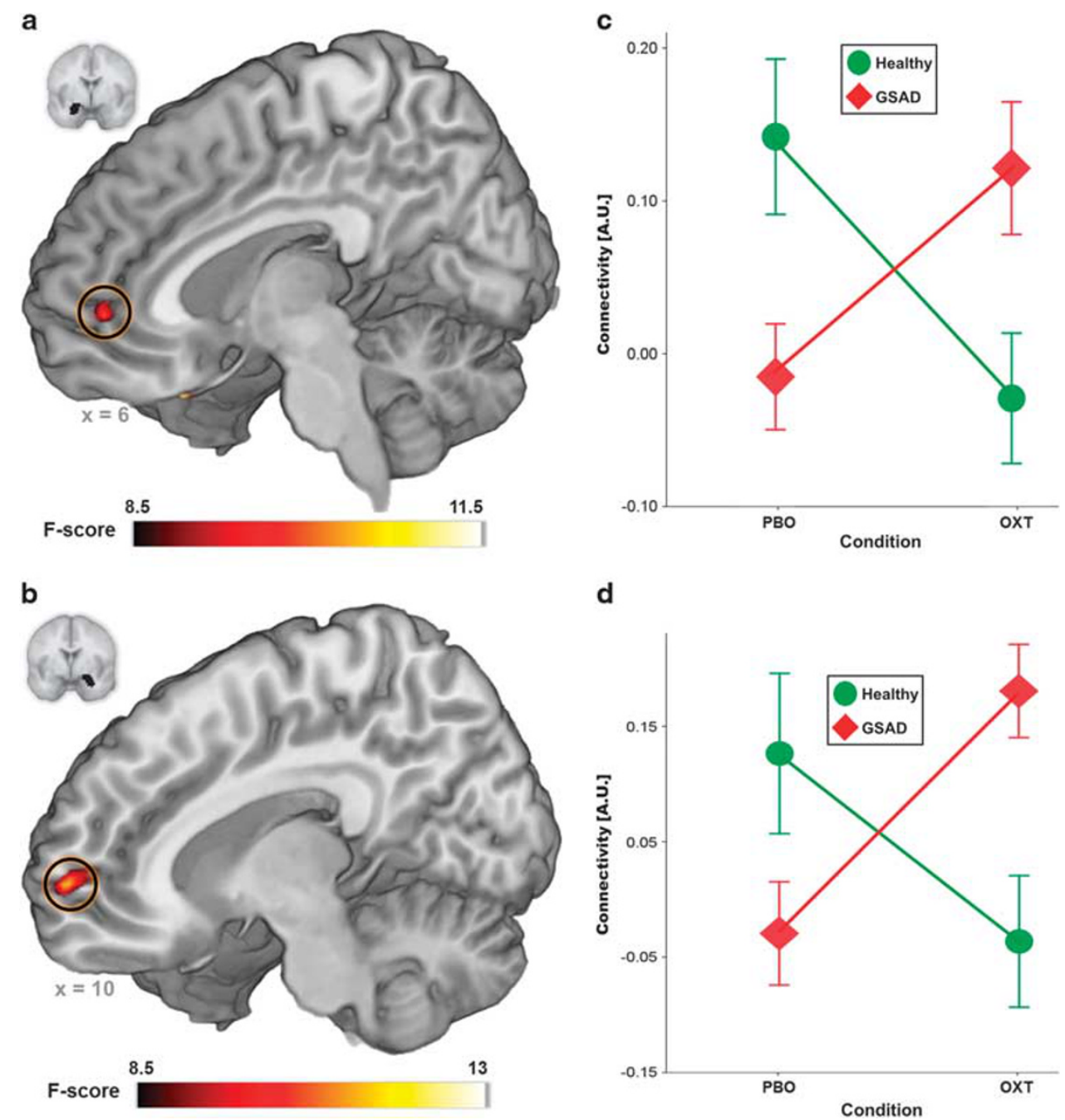

Figure I Resting-state functional connectivity brain maps for left and right amygdala showing: drug-by-group interactions exhibited from left amygdala to ACC (a) and right amygdala to mPFC (b) connectivity. Extracted connectivity strength from left amygdala to ACC (c) and right amygdala to mPFC (d) for each group in session. GSAD, generalized social anxiety disorder group; Healthy, healthy control group; OXT, oxytocin; PBO, placebo.

level in GSAD patients, $t(1,17)=2.06, p=0.055)$, an effect that was observed to be reversed in HCs, $t(1,16)=-2.63$, $p=0.018$; and (3) GSAD patients exhibited significantly greater left amygdala-ACC/mPFC connectivity relative to HCs on OXT alone, $t(1,33)=2.47, p=0.019$ (Figure 1c).

Similarly, a significant drug-by-group interaction in rsFC was observed between the right amygdala and the rostral mPFC (BA 10) (MNI peak $[10,60,4], F(1,65)=12.46$, $p=0.001$ ), encompassing a cluster (volume $=640 \mathrm{~mm}^{3}$ ) that extended posteriorly toward the rostral ACC (BA 32, subpeak at $[6,52,8], F(1,65)=10.15, p=0.003$ ) (Figure $1 \mathrm{~b}$; Table 3). As predicted, post hoc $t$-tests revealed that: (1) GSAD patients exhibited reduced right amygdala-mPFC/ ACC connectivity relative to HCs at baseline (on $\mathrm{PBO}$ ) at trend-level significance, $t(1,33)=1.911, p=0.065$; (2) OXT enhanced right amygdala-mPFC/ACC connectivity significantly in GSAD patients, $t(1,17)=4.889, p<0.001$, an effect opposite in HCs, $t(1,16)=-1.813, p=0.089$; and (3) GSAD patients exhibited greater right amygdala-mPFC/ACC connectivity relative to HCs on OXT alone $t(1,33)=3.135$, $p=0.004$ (Figure 1d).
Significant main effect of drug and main effect of group on resting-state connectivity between left and right amygdala with rest of brain (ie, outside a priori ACC/mPFC regions) are also reported in Tables 2 and 3, respectively. Of note, no between-group differences in movement were observed for either maximum total movement (GSAD vs HC on OXT: $p=0.360$; SAD $v s$ HC on PBO: $p=0.342$ ) or average movement between scans (GSAD vs HC on OXT: $p=0.664$; GSAD $v s$ HC on PBO: $p=0.924)$. Neither group showed session differences in either maximum total (OXT vs PBO in GSAD: $p=0.662$; OXT $v s$ PBO in HC: $p=0.229$ ) or average movement between scans (OXT vs PBO in GSAD: $p=0.570$; OXT $v s$ PBO in HC: $p=0.563)$.

In a post hoc exploratory analysis, we conducted Pearson's correlation coefficient analyses to examine the relationship between amygdala-frontal $\mathrm{rsFC}$ and social anxiety symptom severity as measured by the total score of the LSAS (LSAS-Total) and given the predicted function of OXT 'prosocial' effects, the social situations subscore of the LSAS (LSAS-Social). At baseline (on PBO), there were trend-level negative correlations between the left amygdala- 
Table 2 Whole-Brain Voxel-Wise ANOVA of Resting-State Functional Connectivity with Left Amygdala

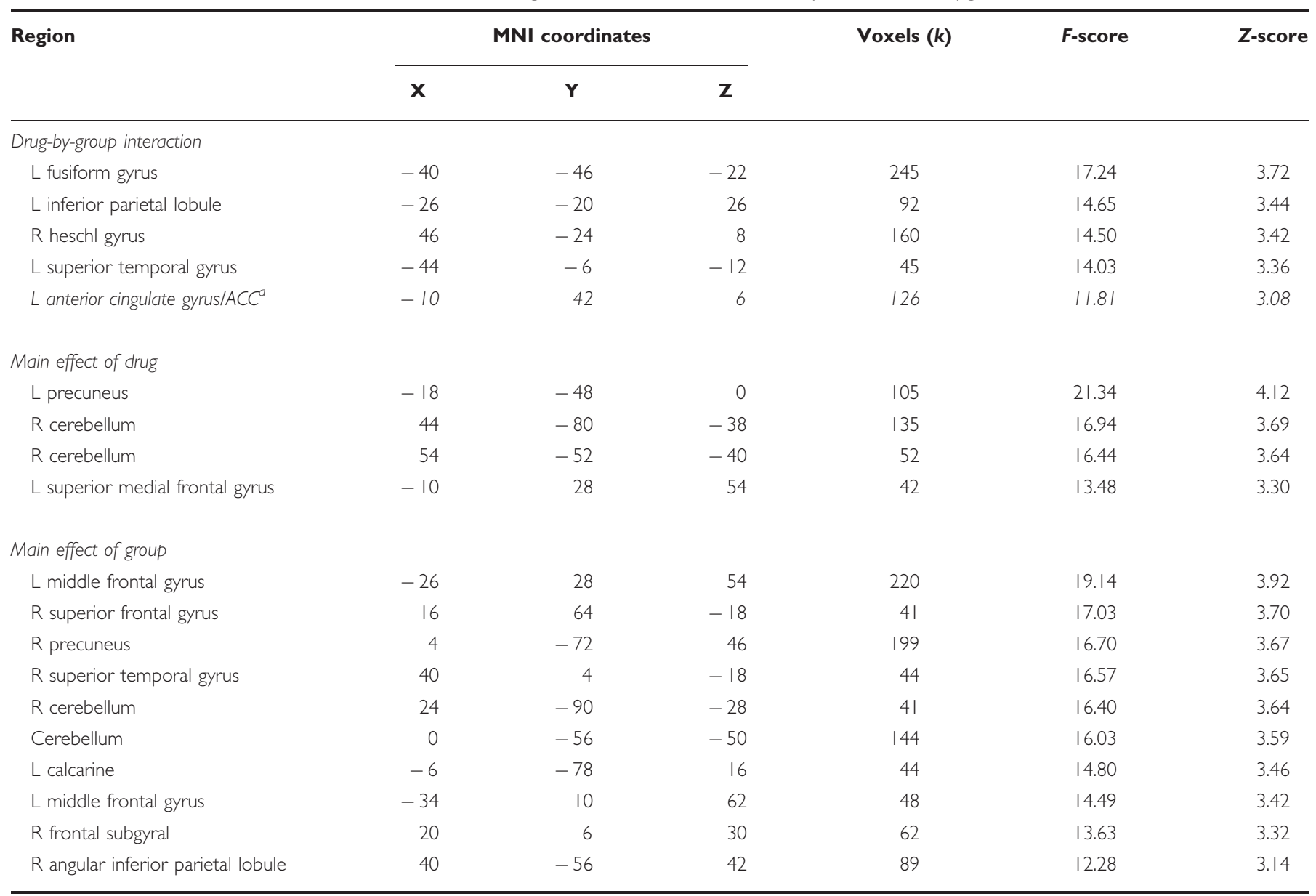

Abbreviations: ACC, anterior cingulate cortex; L, left; MNI, Montreal Neurologic Institute; $R$, right.

Reporting of all clusters exhibiting significance threshold at $p<0.005$ (uncorrected) with a cluster extent threshold of $k$ (number of contiguous voxels) $>40$.

altalics represent a priori areas of interest for significant drug-by-group interaction, corrected for multiple comparisons.

ACC/mPFC connectivity and LSAS-Social score $(r=-0.410$, $p=0.091)$ as well as LSAS-Total scores $(r=-0.422$, $p=0.081$ ) in GSAD patients (Figure $2 \mathrm{a}$ ). The magnitude of change seen on OXT compared with PBO in left amygdalaACC/mPFC connectivity in GSAD patients was also positively correlated with their LSAS-Social score at trend level $(r=0.429, p=0.076)$ such that greater connectivity after OXT administration was observed in those with higher LSAS-Social scores. At baseline (on PBO), there was a significant negative correlation between the right amygdala$\mathrm{mPFC} / \mathrm{ACC}$ connectivity and LSAS-Social score $(r=-0.504$; $p=0.033$ ) in GSAD patients (Figure 2b). A trend-level correlation was also observed between magnitude of change in right amygdala-mPFC/ACC connectivity and LSAS-Social score $(r=0.407 ; p=0.094)$.

\section{DISCUSSION}

To our knowledge, this is the first study of OXT effects on amygdala-frontal connectivity in an anxiety disorder. The findings show that in individuals with GSAD, OXT enhances
rsFC of the left and right amygdala with rostral ACC/mPFC, thereby reversing (ie, normalizing) the reduced amygdalafrontal connectivity observed relative to healthy comparison subjects. Arising from significant drug-by-group interactions, these focal effects of OXT in similar frontal areas (ACC and $\mathrm{mPFC}$ representing BAs 32 and 10, respectively) from both left and right amygdala seed regions are noteworthy. We also found that in GSAD subjects, higher social anxiety correlates with lower amygdala-ACC/mPFC connectivity on PBO and that higher social anxiety also correlated with greater enhancement in amygdala-frontal connectivity induced by OXT. These findings shed some light on a putative neural target and mechanism for OXT's effects.

The current results show that the resting-state connectivity from left and right amygdala to specific regions of the frontal cortex (ACC and mPFC/rACC) is reduced in GSAD patients when compared with $\mathrm{HCs}$ at baseline (on PBO). These findings are consistent with two prior papers from independent cohorts (Hahn et al, 2011; Prater et al, 2013). Our findings of decreased amygdala mPFC/rACC connectivity are further strengthened by the finding of significant negative correlations between anxiety severity in social 
Table 3 Whole-Brain Voxel-Wise ANOVA of Resting-State Functional Connectivity with Right Amygdala

\begin{tabular}{|c|c|c|c|c|c|c|}
\hline \multirow[t]{2}{*}{ Region } & \multicolumn{3}{|c|}{$\begin{array}{c}\text { MNI } \\
\text { coordinates }\end{array}$} & \multirow[t]{2}{*}{$\begin{array}{c}\text { Voxels } \\
\text { (k) }\end{array}$} & \multirow[t]{2}{*}{$\begin{array}{c}\text { F- } \\
\text { score }\end{array}$} & \multirow[t]{2}{*}{$\begin{array}{c}\text { Z- } \\
\text { score }\end{array}$} \\
\hline & $\mathbf{x}$ & $\mathbf{Y}$ & $\mathbf{Z}$ & & & \\
\hline \multicolumn{7}{|l|}{ Drug-by-group interaction } \\
\hline L superior temporal gyrus & -66 & -42 & 18 & 67 & 18.42 & 3.85 \\
\hline L inferior temporal gyrus & -44 & -12 & -46 & 83 & 17.63 & 3.76 \\
\hline R superior occipital lobe & 20 & -88 & 36 & 70 & 13.22 & 3.26 \\
\hline$R$ medial frontal gyrus/mPFC & 10 & 60 & 4 & 80 & 12.46 & 3.17 \\
\hline $\mathrm{L}$ calcarine & -4 & -90 & -12 & 43 & 12.42 & 3.16 \\
\hline \multicolumn{7}{|l|}{ Main effect of drug } \\
\hline L inferior temporal gyrus & -50 & -6 & -44 & 111 & 18.46 & 3.85 \\
\hline R precentral gyrus & 40 & -6 & 40 & 64 & 17.16 & 3.72 \\
\hline L cerebrospinal fluid & -16 & -38 & 18 & 98 & 17.12 & 3.71 \\
\hline R Inferior Parietal Lobule & 38 & -46 & 28 & 53 & 15.19 & 3.50 \\
\hline $\mathrm{R}$ inferior parietal lobule & 60 & -26 & 34 & 47 & 14.08 & 3.37 \\
\hline R sublocar & 24 & -30 & 14 & 40 & 13.32 & 3.28 \\
\hline $\mathrm{R}$ middle cingulum & 4 & -2 & 40 & 45 & 11.28 & 3.01 \\
\hline \multicolumn{7}{|l|}{ Main effect of group } \\
\hline R superior frontal gyrus & 18 & 50 & 12 & 120 & 23.65 & 4.32 \\
\hline$L$ frontal orbital gyrus & -12 & 48 & -26 & 64 & 18.50 & 3.85 \\
\hline L brainstem & -6 & -26 & -36 & 43 & 16.22 & 3.61 \\
\hline $\mathrm{R}$ inferior frontal gyrus & 36 & 26 & 14 & 123 & 15.99 & 3.59 \\
\hline R paracentral lobule & 6 & -32 & 74 & 93 & 15.80 & 3.57 \\
\hline $\mathrm{R}$ medial frontal gyrus & 18 & 46 & -6 & 63 & 13.76 & 3.33 \\
\hline
\end{tabular}

Abbreviations: L, left; MNI, Montreal Neurologic Institute; mPFC, medial prefrontal cortex; $\mathrm{R}$, right.

Reporting of all clusters exhibiting significance threshold at $p<0.005$ (uncorrected) with a cluster extent threshold of $k$ (number of contiguous voxels) $>40$

altalics represent a priori areas of interest for significant drug-by-group interaction, corrected for multiple comparisons.

situations (LSAS-Social score) and ACC/mPFC connectivity with both left and right amygdala-ACC connectivity at baseline (on PBO). This shows that GSAD patients with relatively more severe levels of social interactional anxiety have lower amygdala-ACC/mPFC connectivity than those with less severe social interactional anxiety. Other studies have suggested that the rACC has a regulatory role via topdown control of amygdala reactivity in the processing of negative emotions (Bishop et al, 2004; Etkin and Schatzberg, 2011). It has been posited that specifically the rostral ACC and amygdala dynamically interact to regulate the processing of social signals of threat (Kim et al, 2011) and resolving interference from social threat (Etkin et al, 2006), which are processes highly relevant in GSAD (Goldin et al, 2009; Klumpp et al, 2012). Abnormalities involving the $\mathrm{mPFC} / \mathrm{ACC}$ in GSAD patients are not limited to resting-state studies and have been shown in tasks involving emotion appraisal. This suggests that the $\mathrm{rACC} / \mathrm{mPFC}$ may have a
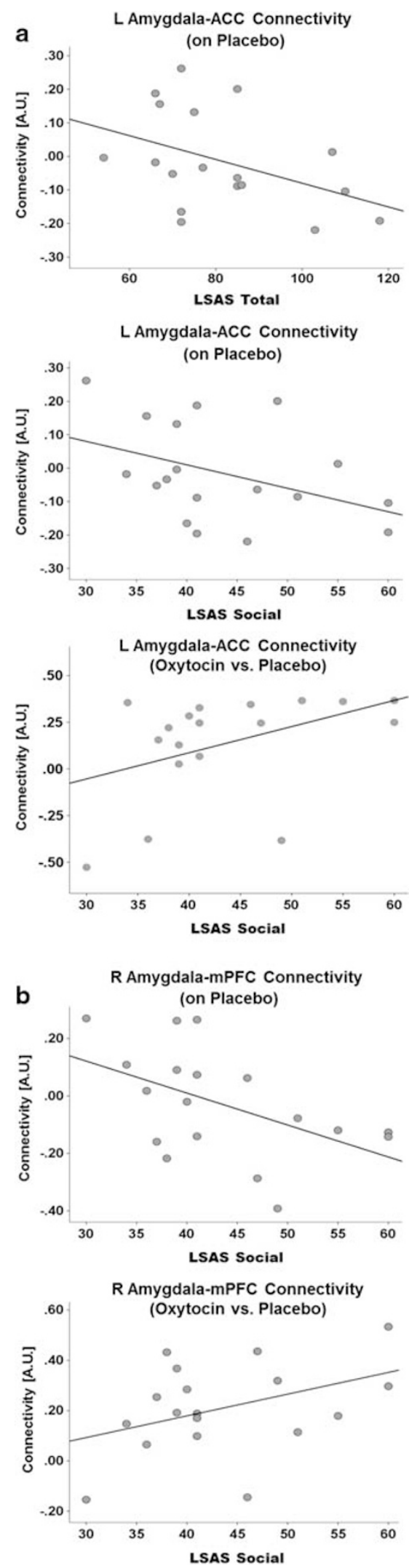

Figure 2 Scatter plot of correlation between extent of resting-state functional connectivity from left amygdala to ACC (a) and right amygdala to mPFC (b) and severity of social anxiety as indexed by the Liebowitz Social Anxiety Scale (LSAS) for the total score or social situations subscale score. 
role in regulating the processing of emotions, and that this role may be compromised in GSAD patients (Klumpp et al, $2013 \mathrm{~b})$. The reduced left amygdala-rACC connectivity we found in GSAD patients at resting state may indicate an abnormal threat-emotion processing circuit in these patients at baseline and may be a marker of the pathophysiology of GSAD at rest and during threat perception (Prater et al, 2013).

Importantly, the findings also show that acute OXT treatment significantly increases $\mathrm{rsFC}$ of left and right amygdala to ACC/mPFC in GSAD patients. Remarkably, this enhanced connectivity of amygdala bilaterally under the influence of OXT was seen in a similar, but focal area of the frontal cortex in contiguous BAs 32 and 10, both previously known to interact with the amygdala during threat and emotion regulation (Banks et al, 2007; Etkin et al, 2006; Wager et al, 2008). Our findings of enhanced connectivity suggest that OXT may have a role in ameliorating abnormalities associated with emotion regulation pathways in GSAD observed at rest (Prater et al, 2013), suggesting a neural mechanism by which OXT may exert its effects acutely. Interestingly, some evidence has suggested that OXT can be an effective treatment for GSAD (Guastella et al, 2009). The current findings and previous studies including our own provide a neurobiological mechanism for a potential behavioral effect. We had previously shown that acute OXT exerted effects on the amygdala and mPFC/ACC during the processing of threat and other negative emotions (Labuschagne et al, 2010, 2011). Both the amygdala and rostral ACC/mPFC are putative neural 'targets' of psychosocial and pharmacological treatments known to be effective in GSAD (Evans et al, 2009; Goldin et al, 2013; Klumpp et al, 2013a; Phan et al, 2013). Furthermore, our correlation analysis found that in GSAD patients, the magnitude of change induced by OXT (from $\mathrm{PBO}$ ) in left amygdala-ACC/mPFC is correlated with the severity of social interactional anxiety such that those with the highest levels of anxiety may benefit the most from OXT treatment. This suggest that OXT's prosocial and/or anti-anxiety effects may have its greatest effects in GSAD individuals who exhibit the most attenuated amygdala-ACC/mPFC connectivity at baseline.

We had previously shown that OXT enhances amygdala connectivity to rostral ACC at rest in healthy volunteers (Sripada et al, 2013). Interestingly, Zink and MeyerLindenberg (2012) found that vasopressin, which is thought to have an effect opposite to OXT by enhancing fear and anxiety (Viviani and Stoop, 2008), decreased amygdala to medial prefrontal connectivity in group of healthy volunteers. Augmentation of connectivity between ACC and amygdala suggests increased interaction between these regions under the influence of OXT. Therefore, this effect of OXT may possibly be due to its ability to facilitate enhanced interaction between two key brain regions involved in the evaluation of social stimuli. Prior studies including those involving GSAD patients have shown OXT effects on these two brain regions. In two recent task-based studies, we showed that OXT attenuates heightened activity in the amygdala of GSAD patients after viewing fearful faces (Labuschagne et al, 2010) and in the mPFC/ACC after they view sad faces (Labuschagne et al, 2011). Our findings therefore might provide a mechanistic explanation of the underlying processes involved in the dampening of amygdala and medial frontal hyperactivity as has been reported previously. These observations of enhanced connectivity may be pertinent in other disorders associated with amygdala-mPFC disruption and associated social dysfunction like depression, autism, and schizophrenia. Indeed, OXT has been suggested as a novel therapeutic agent to enhance socialization in autism (Farmer et al, 2013). This therapeutic benefit may be due to improved amygdalamPFC connectivity.

Resting-state connectivity analysis assesses correlations between spontaneous, slow $(<0.1 \mathrm{~Hz})$ fluctuations in brain activation-regions exhibiting high degrees of coherence during the resting state are thought to be functionally interconnected in terms of flow of neural information and/or mutual regulation (Fox and Raichle, 2007). This approach helps identifying the brain regions coupled with each other before task. Amygdala and ACC have been postulated to be essential components of the brain's 'salience network', whereby it has a role in evaluating approaching stimuli and have been considered to be critical nodes for social-affective functioning. Deficits in amygdala-ACC connectivity have been suggested to have a role in the brain models of GSAD. There have been suggestions of possible tonic and phasic alterations in this network (Prater et al, 2013). While OXT appears to enhance resting-state connectivity in GSAD patients, it would be interesting to see whether this would also occur during tasks pertaining to emotion processing and regulation. As this study assessed connectivity at a resting state, further studies are needed to assess effects of OXT on connectivity during emotion processing and regulation.

The current findings should be considered in the context of several limitations. First, we assessed amygdala connectivity during the resting state. Future studies should ascertain whether OXT affects connectivity during socioemotion processing and regulation tasks and to explore whether this would be associated with social and/or emotional functioning. Second, OXT has been shown to have differential effects on emotional responding in males and females (Domes et al, 2010). Our study explored OXT's effect only in males at rest, thus further studies are needed to see whether our findings would generalize to women. Third, this study does not permit identification of the neurochemical mechanisms by which intranasal OXT affects resting-state amygdala connectivity. Previous studies have shown that OXT receptors in the amygdala potentiate GABAergic inhibition (ie, Huber et al, 2005; Viviani et al, 2011). Whether this mechanism has a role in OXT modulation of connectivity of the amygdala is however currently unknown. Fourth, although the current sample is one of the largest acute pharmaco-fMRI studies of an anxiety disordered patient population, our findings are limited by the small sample size, introducing greater risk for Type II errors, which is also contributed to by the shorter scan duration ( $3 \mathrm{~min} 20 \mathrm{~s}$ ), relative to other resting-state studies (Fox and Raichle, 2007); however, expected amygdala connectivity patterns to $\mathrm{mPFC}$, cingulate cortex, and insula were still evident on the resting-state scans in HCs on PBO session (data not shown). It should be noted the current design does not allow us to disambiguate the effect of PBO and thus the 'baseline' measure may not represent a true 
baseline and would more accurately be interpreted as functional connectivity under PBO. The design also involved the resting-state scans typically occurring after the emotional faces processing scans, which could exert effects on the resting-state condition; however, this is partly mitigated by our PBO-controlled design. Finally, while acute PBOcontrolled pharmacological fMRI studies provide insight into the effects OXT on rsFC, interpretations about mechanisms are limited and additional research is needed to determine how these connectivity patterns relate to clinical outcomes or to underlying dysfunctional processes, and to measure test-retest reliability.

In conclusion, we coupled resting-state fMRI with an acute pharmacological OXT challenge to examine the effects of OXT on amygdala-frontal connectivity at rest in patients with GSAD. We found that OXT enhanced functional connectivity between amygdala and specific areas of the frontal cortex (ACC and mPFC) in our patients with GSAD, which constitute the neural circuit known for its role in social threat processing and emotion regulation. These findings add to our understanding of the neural mechanisms by which OXT may have a role in the pathophysiology and/or treatment of social anxiety disorder.

\section{FUNDING AND DISCLOSURE}

PJN was an employee of GSK and UCB Pharma during the conduct of the study but received no funding from both companies for the study. The authors declare no conflict of interests.

\section{ACKNOWLEDGEMENTS}

This research was funded by an Independent Investigator Award to PJN from the Brain and Behavior Research Foundation (formally National Alliance for Research in Schizophrenia and Depression [NARSAD]). AGW was supported by an Australian Rotary Health fellowship.

\section{REFERENCES}

Adolphs R (2009). The social brain: neural basis of social knowledge. Annu Rev Psychol 60: 693-716.

Amir N, Klumpp H, Elias J, Bedwell JS, Yanasak N, Miller LS (2005). Increased activation of the anterior cingulate cortex during processing of disgust faces in individuals with social phobia. Biol Psychiatry 57: 975-981.

Banks SJ, Eddy KT, Angstadt M, Nathan PJ, Phan KL (2007). Amygdala-frontal connectivity during emotion regulation. Soc Cogn Affect Neurosci 2: 303-312.

Bartz JA, Zaki J, Bolger N, Ochsner KN (2011). Social effects of oxytocin in humans: context and person matter. Trends Cogn Sci 15: 301-309.

Beck AT, Steer RA, Ball R, Ranieri WF (1996). Comparison of Beck Depression Inventories-IA and-II in psychiatric outpatients. J Pers Assess 67: 588-597.

Behzadi Y, Restom K, Liau J, Liu TT (2007). A component based noise correction method (CompCor) for BOLD and perfusion based fMRI. Neuroimage 37: 90-101.

Bethlehem RA, van Honk J, Auyeung B, Baron-Cohen S (2013). Oxytocin, brain physiology, and functional connectivity: a review of intranasal oxytocin fMRI studies. Psychoneuroendocrinology 38: 962-974.
Bielsky IF, Young LJ (2004). Oxytocin, vasopressin, and social recognition in mammals. Peptides 25: 1565-1574.

Bishop SJ, Duncan J, Lawrence AD (2004). State anxiety modulation of the amygdala response to unattended threatrelated stimuli. J Neurosci 24: 10364-10368.

Carter CS, Grippo AJ, Pournajafi-Nazarloo H, Ruscio MG, Porges SW (2008). Oxytocin, vasopressin and sociality. Prog Brain Res 170: $331-336$.

Davidson RJ, Marshall JR, Tomarken AJ, Henriques JB (2000). While a phobic waits: regional brain electrical and autonomic activity in social phobics during anticipation of public speaking. Biol Psychiatry 47: 85-95.

Domes G, Heinrichs M, Gläscher J, Büchel C, Braus DF, Herpertz SC (2007). Oxytocin attenuates amygdala responses to emotional faces regardless of valence. Biol Psychiatry 62: 1187-1190.

Domes G, Lischke A, Berger C, Grossmann A, Hauenstein K, Heinrichs $\mathrm{M}$ et al (2010). Effects of intranasal oxytocin on emotional face processing in women. Psychoneuroendocrinology 35: 83-93.

Ehrlich I, Humeau Y, Grenier F, Ciocchi S, Herry C, Lüthi A (2009). Amygdala inhibitory circuits and the control of fear memory. Neuron 62: 757-771.

Etkin A, Egner T, Peraza DM, Kandel ER, Hirsch J (2006). Resolving emotional conflict: a role for the rostral anterior cingulate cortex in modulating activity in the amygdala. Neuron 51: 871-882.

Etkin A, Schatzberg AF (2011). Common abnormalities and disorder-specific compensation during implicit regulation of emotional processing in generalized anxiety and major depressive disorders. Am J Psychiatry 168: 968-978.

Evans KC, Simon NM, Dougherty DD, Hoge EA, Worthington JJ, Chow C et al (2009). A PET study of tiagabine treatment implicates ventral medial prefrontal cortex in generalized social anxiety disorder. Neuropsychopharmacology 34: 390-398.

Evans KC, Wright CI, Wedig MM, Gold AL, Pollack MH, Rauch SL (2008). A functional MRI study of amygdala responses to angry schematic faces in social anxiety disorder. Depress Anxiety 25: 496-505.

Farmer C, Thurm A, Grant P (2013). Pharmacotherapy for the core symptoms in autistic disorder: current status of the research. Drugs 73: 303-314.

Fox MD, Raichle ME (2007). Spontaneous fluctuations in brain activity observed with functional magnetic resonance imaging. Nat Rev Neurosci 8: 700-711.

Ghashghaei HT, Hilgetag CC, Barbas H (2007). Sequence of information processing for emotions based on the anatomic dialogue between prefrontal cortex and amygdala. Neuroimage 34: 905-923.

Gimpl G, Fahrenholz F (2001). The oxytocin receptor system: structure, function, and regulation. Physiol Rev 81: 629-683.

Goldin PR, Manber T, Hakimi S, Canli T, Gross JJ (2009). Neural bases of social anxiety disorder: emotional reactivity and cognitive regulation during social and physical threat. Arch Gen Psychiatry 66: 170-180.

Goldin PR, Ziv M, Jazaieri H, Hahn K, Heimberg R, Gross JJ (2013). Impact of cognitive behavioral therapy for social anxiety disorder on the neural dynamics of cognitive reappraisal of negative self-beliefs: randomized clinical trial. JAMA Psychiatry 70: $1048-1056$.

Guastella AJ, Howard AL, Dadds MR, Mitchell P, Carson DS (2009). A randomized controlled trial of intranasal oxytocin as an adjunct to exposure therapy for social anxiety disorder. Psychoneuroendocrinology 34: 917-923.

Hahn A, Stein P, Windischberger C, Weissenbacher A, Spindelegger C, Moser E et al (2011). Reduced resting-state functional connectivity between amygdala and orbitofrontal cortex in social anxiety disorder. Neuroimage 56: 881-889.

Huber D, Veinante P, Stoop R (2005). Vasopressin and oxytocin excite distinct neuronal populations in the central amygdala. Science 308: 245-248. 
Kim MJ, Loucks RA, Palmer AL, Brown AC, Solomon KM, Marchante AN et al (2011). The structural and functional connectivity of the amygdala: from normal emotion to pathological anxiety. Behav Brain Res 223: 403-410.

Kirsch P, Esslinger C, Chen Q, Mier D, Lis S, Siddhanti S et al (2005). Oxytocin modulates neural circuitry for social cognition and fear in humans. J Neurosci 25: 11489-11493.

Klumpp H, Angstadt M, Phan KL (2012). Insula reactivity and connectivity to anterior cingulate cortex when processing threat in generalized social anxiety disorder. Biol Psychol 89: 273-276.

Klumpp H, Fitzgerald DA, Phan KL (2013a). Neural predictors and mechanisms of cognitive behavioral therapy on threat processing in social anxiety disorder. Prog Neuropsychopharmacol Biol Psychiatry 45: 83-91.

Klumpp H, Post D, Angstadt M, Fitzgerald DA, Phan KL (2013b). Anterior cingulate cortex and insula response during indirect and direct processing of emotional faces in generalized social anxiety disorder. Biol Mood Anxiety Disord 3: 7.

Labuschagne I, Phan KL, Wood A, Angstadt M, Chua P, Heinrichs $\mathrm{M}$ et al (2010). Oxytocin attenuates amygdala reactivity to fear in generalized social anxiety disorder. Neuropsychopharmacology 35: 2403-2413.

Labuschagne I, Phan KL, Wood A, Angstadt M, Chua P, Heinrichs $M$ et al (2011). Medial frontal hyperactivity to sad faces in generalized social anxiety disorder and modulation by oxytocin. Int J Neuropsychopharmacol 1-14.

Liao W, Chen H, Feng Y, Mantini D, Gentili C, Pan Z et al (2010). Selective aberrant functional connectivity of resting state networks in social anxiety disorder. Neuroimage 52: 1549-1558.

Lieberman MD, Cunningham WA (2009). Type I and Type II error concerns in fMRI research: re-balancing the scale. Soc $\operatorname{Cog} n$ Affect Neurosci 4: 423-428.

Liebowitz MR (1987). Social phobia. Mod Probl Pharmacopsychiatry 22: 141-173.

Petrovic P, Kalisch R, Singer T, Dolan RJ (2008). Oxytocin attenuates affective evaluations of conditioned faces and amygdala activity. J Neurosci 28: 6607-6615.

Phan KL, Coccaro EF, Angstadt M, Kreger KJ, Mayberg HS, Liberzon I et al (2013). Corticolimbic brain reactivity to social signals of threat before and after sertraline treatment in generalized social phobia. Biol Psychiatry 73: 329-336.

Phan KL, Fitzgerald DA, Nathan PJ, Tancer ME (2006). Association between amygdala hyperactivity to harsh faces and severity of social anxiety in generalized social phobia. Biol Psychiatry 59: 424-429.

Prater KE, Hosanagar A, Klumpp H, Angstadt M, Phan KL (2013). Aberrant amygdala-frontal cortex connectivity during perception of fearful faces and at rest in generalized social anxiety disorder. Depress Anxiety 30: 234-241.

Spielberger CD (1983). Manual for the State-Trait Anxiety Inventory (form Y). Consulting Psychologists Press: Palo Alto, CA.

Sripada CS, Phan KL, Labuschagne I, Welsh R, Nathan PJ, Wood AG (2013). Oxytocin enhances resting-state connectivity between amygdala and medial frontal cortex. Int J Neuropsychopharmacol 16: 255-260.

Steer RA, Beck AT (1997). Beck anxiety inventory. Evaluating Stress: A Book of Resources. Scarecrow Press: Lanham, MD, PP 23-40.

Stein MB, Goldin PR, Sareen J, Zorrilla L, Brown GG (2002). Increased amygdala activation to angry and contemptuous faces in generalized social phobia. Arch Gen Psychiatry 59: 1027-1034.

Stein MB, Stein DJ (2008). Social anxiety disorder. Lancet 371: $1115-1125$.

Tzourio-Mazoyer N, Landeau B, Papathanassiou D, Crivello F, Etard O, Delcroix N et al (2002). Automated anatomical labeling of activations in SPM using a macroscopic anatomical parcellation of the MNI MRI single-subject brain. Neuroimage 15: 273-289.

Viviani D, Charlet A, Burg E, van den, Robinet C, Hurni N, Abatis $\mathrm{M}$ et al (2011). Oxytocin selectively gates fear responses through distinct outputs from the central amygdala. Science 333: 104-107.

Viviani D, Stoop R (2008). Opposite effects of oxytocin and vasopressin on the emotional expression of the fear response. Prog Brain Res 170: 207-218.

Wager TD, Davidson ML, Hughes BL, Lindquist MA, Ochsner KN (2008). Prefrontal-subcortical pathways mediating successful emotion regulation. Neuron 59: 1037-1050.

Whitfield-Gabrieli S, Nieto-Castanon A (2012). Conn: a functional connectivity toolbox for correlated and anticorrelated brain networks. Brain Connect 2: 125-141.

World Health Organization (1997). Composite International Diagnostic Interview, Version 2.1. World Health Organization: Geneva, Switzerland.

Zink CF, Meyer-Lindenberg A (2012). Human neuroimaging of oxytocin and vasopressin in social cognition. Horm Behav 61: 400-409. 